\title{
JOHANN FAULHABER AND SUMS OF POWERS
}

\author{
DONALD E. KNUTH
}

Dedicated to the memory of D. H. Lehmer

\begin{abstract}
Early 17th-century mathematical publications of Johann Faulhaber contain some remarkable theorems, such as the fact that the $r$-fold summation of $1^{m}, 2^{m}, \ldots, n^{m}$ is a polynomial in $n(n+r)$ when $m$ is a positive odd number. The present paper explores a computation-based approach by which Faulhaber may well have discovered such results, and solves a 360 -year-old riddle that Faulhaber presented to his readers. It also shows that similar results hold when we express the sums in terms of central factorial powers instead of ordinary powers. Faulhaber's coefficients can moreover be generalized to noninteger exponents, obtaining asymptotic series for $1^{\alpha}+2^{\alpha}+\cdots+n^{\alpha}$ in powers of $n^{-1}(n+1)^{-1}$.
\end{abstract}

\section{INTRODUCTION}

Johann Faulhaber of Ulm (1580-1635), founder of a school for engineers early in the 17th century, loved numbers. His passion for arithmetic and algebra led him to devote a considerable portion of his life to the computation of formulas for the sums of powers, significantly extending all previously known results. Indeed, he may well have carried out more computing than anybody else in Europe during the first half of the 17th century. His greatest mathematical achievements appear in a booklet entitled Academia Algebra (written in German in spite of its Latin title), published in Augsburg, 1631 [2]. Here we find, for example, the following formulas for sums of odd powers:

$$
\begin{aligned}
1^{1}+2^{1}+\cdots+n^{1} & =N, \quad N=\left(n^{2}+n\right) / 2 ; \\
1^{3}+2^{3}+\cdots+n^{3} & =N^{2} ; \\
1^{5}+2^{5}+\cdots+n^{5} & =\left(4 N^{3}-N^{2}\right) / 3 ; \\
1^{7}+2^{7}+\cdots+n^{7} & =\left(12 N^{4}-8 N^{3}+2 N^{2}\right) / 6 ; \\
1^{9}+2^{9}+\cdots+n^{9} & =\left(16 N^{5}-20 N^{4}+12 N^{3}-3 N^{2}\right) / 5 ; \\
1^{11}+2^{11}+\cdots+n^{11} & =\left(32 N^{6}-64 N^{5}+68 N^{4}-40 N^{3}+10 N^{2}\right) / 6 ; \\
1^{13}+2^{13}+\cdots+n^{13} & =\left(960 N^{7}-2800 N^{6}+4592 N^{5}-4720 N^{4}\right. \\
+ & \left.+2764 N^{3}-691 N^{2}\right) / 105 ; \\
1^{15}+2^{15}+\cdots+n^{15}= & \left(192 N^{8}-768 N^{7}+1792 N^{6}-2816 N^{5}\right. \\
& \left.+2872 N^{4}-1680 N^{3}+420 N^{2}\right) / 12 ;
\end{aligned}
$$

Received by the editor July 27, 1992.

1991 Mathematics Subject Classification. Primary 11B83, 01A45; Secondary 11B37, 30E15. 


$$
\begin{aligned}
1^{17}+2^{17}+\cdots+n^{17}= & \left(1280 N^{9}-6720 N^{8}+21120 N^{7}-46880 N^{6}\right. \\
& \left.+72912 N^{5}-74220 N^{4}+43404 N^{3}-10851 N^{2}\right) / 45 .
\end{aligned}
$$

Other mathematicians had studied $\Sigma n^{1}, \Sigma n^{2}, \ldots, \Sigma n^{7}$, and he had previously gotten as far as $\Sigma n^{12}$; but the sums had always previously been expressed as polynomials in $n$, not $N$.

Faulhaber begins his book by simply stating these novel formulas and proceeding to expand them into the corresponding polynomials in $n$. Then he verifies the results when $n=4, N=10$. But he gives no clues about how he derived the expressions; he states only that the leading coefficient in $\Sigma n^{2 m-1}$ will be $2^{m-1} / m$, and that the trailing coefficients will have the form $4 \alpha_{m} N^{3}-\alpha_{m} N^{2}$ when $m \geq 3$.

Faulhaber believed that similar polynomials in $N$, with alternating signs, would continue to exist for all $m$, but he may not really have known how to prove such a theorem. In his day, mathematics was treated like all other sciences; an observed phenomenon was considered to be true if it was supported by a large body of evidence. A rigorous proof of Faulhaber's assertion was first published by Jacobi in 1834 [6]. A. W. F. Edwards showed recently how to obtain the coefficients by matrix inversion [1], based on another proof given by L. Tits in 1923 [8]. But none of these proofs use methods that are very close to those known in 1631.

Faulhaber went on to consider sums of sums. Let us write $\Sigma^{r} n^{m}$ for the $r$-fold summation of $m$ th powers from 1 to $n$; thus,

$$
\Sigma^{0} n^{m}=n^{m} ; \quad \Sigma^{r+1} n^{m}=\Sigma^{r} 1^{m}+\Sigma^{r} 2^{m}+\cdots+\Sigma^{r} n^{m} .
$$

He discovered that $\Sigma^{r} n^{2 m}$ can be written as a polynomial in the quantity

$$
N_{r}=\left(n^{2}+r n\right) / 2 \text {, }
$$

times $\Sigma^{r} n^{2}$. For example, he gave the formulas

$$
\begin{aligned}
& \Sigma^{2} n^{4}=\left(4 N_{2}-1\right) \Sigma^{2} n^{2} / 5 ; \\
& \Sigma^{3} n^{4}=\left(4 N_{3}-1\right) \Sigma^{3} n^{2} / 7 ; \\
& \Sigma^{4} n^{4}=\left(6 N_{4}-1\right) \Sigma^{4} n^{2} / 14 ; \\
& \Sigma^{6} n^{4}=\left(4 N_{6}+1\right) \Sigma^{6} n^{2} / 15 ; \\
& \Sigma^{2} n^{6}=\left(6 N_{2}^{2}-5 N_{2}+1\right) \Sigma^{2} n^{2} / 7 ; \\
& \Sigma^{3} n^{6}=\left(10 N_{3}^{2}-10 N_{3}+1\right) \Sigma^{3} n^{2} / 21 ; \\
& \Sigma^{4} n^{6}=\left(4 N_{4}^{2}-4 N_{4}-1\right) \Sigma^{4} n^{2} / 14 ; \\
& \Sigma^{2} n^{8}=\left(16 N_{2}^{3}-28 N_{2}^{2}+18 N_{2}-3\right) \Sigma^{2} n^{2} / 15 .
\end{aligned}
$$

He also gave similar formulas for odd exponents, factoring out $\Sigma^{r} n^{1}$ instead of $\Sigma^{r} n^{2}$ :

$$
\begin{aligned}
& \Sigma^{2} n^{5}=\left(8 N_{2}^{2}-2 N_{2}-1\right) \Sigma^{2} n^{1} / 14 ; \\
& \Sigma^{2} n^{7}=\left(40 N_{2}^{3}-40 N_{2}^{2}+6 N_{2}+6\right) \Sigma^{2} n^{1} / 60 .
\end{aligned}
$$

And he claimed that, in general, $\Sigma^{r} n^{m}$ can be expressed as a polynomial in $N_{r}$ times either $\Sigma^{r} n^{2}$ or $\Sigma^{r} n^{1}$, depending on whether $m$ is even or odd.

Faulhaber had probably verified this remarkable theorem in many cases including $\Sigma^{11} n^{6}$, because he exhibited a polynomial in $n$ for $\Sigma^{11} n^{6}$ that would 
have been quite difficult to obtain by repeated summation. His polynomial, which has the form

$$
\frac{6 n^{17}+561 n^{16}+\cdots+1021675563656 n^{5}+\cdots-96598656000 n}{2964061900800}
$$

turns out to be absolutely correct, according to calculations with a modern computer. (The denominator is $17 ! / 120$. One cannot help thinking that nobody has ever checked these numbers since Faulhaber himself wrote them down, until today.)

Did he, however, know how to prove his claim, in the sense that 20th century mathematicians would regard his argument as conclusive? He may in fact have known how to do so, because there is an extremely simple way to verify the result using only methods that he would have found natural.

\section{REFLECTIVE FUNCTIONS}

Let us begin by studying an elementary property of functions defined on the integers. We will say that the function $f(x)$ is $r$-reflective if

$$
f(x)=f(y) \text { whenever } x+y+r=0 \text {; }
$$

and it is anti-r-reflective if

$$
f(x)=-f(y) \text { whenever } x+y+r=0 .
$$

The values of $x, y, r$ will be assumed to be integers for simplicity. When $r=0$, reflective functions are even, and anti-reflective functions are odd. Notice that $r$-reflective functions are closed under addition and multiplication; moreover, the product of two anti-r-reflective functions is $r$-reflective.

Given a function $f$, we define its backward difference $\nabla f$ in the usual way:

$$
\nabla f(x)=f(x)-f(x-1) .
$$

It is now easy to verify a simple basic fact.

Lemma 1. If $f$ is r-reflective, then $\nabla f$ is anti- $(r-1)$-reflective. If $f$ is anti-rreflective, then $\nabla f$ is $(r-1)$-reflective.

Proof. If $x+y+(r-1)=0$, then $x+(y-1)+r=0$ and $(x-1)+y+r=0$. Thus $f(x)= \pm f(y-1)$ and $f(x-1)= \pm f(y)$ when $f$ is $r$-reflective or anti-r-reflective.

Faulhaber almost certainly knew this lemma, because [2, folio D.iii recto] presents a table of $n^{8}, \nabla n^{8}, \ldots, \nabla^{8} n^{8}$ in which the reflection phenomenon is clearly apparent. He states that he has constructed "grosse Tafeln," but that this example should be "alles gnugsam vor Augen sehen und auf höhere quantiteten [exponents] continuiren könde."

The converse of Lemma 1 is also true, if we are careful. Let us define $\Sigma$ as an inverse to the $\nabla$ operator:

$$
\Sigma f(n)= \begin{cases}C+f(1)+\cdots+f(n), & \text { if } n \geq 0 \\ C-f(0)-\cdots-f(n+1), & \text { if } n<0\end{cases}
$$

Here $C$ is an unspecified constant, which we will choose later; whatever its value, we have

$$
\nabla \Sigma f(n)=\Sigma f(n)-\Sigma f(n-1)=f(n)
$$

for all $n$. 
Lemma 2. If $f$ is r-reflective, there is a unique $C$ such that $\Sigma f$ is anti- $(r+1)$ reflective. If $f$ is anti-r-reflective, then $\Sigma f$ is $(r+1)$-reflective for all $C$.

Proof. If $r$ is odd, $\Sigma f$ can be anti- $(r+1)$-reflective only if $C$ is chosen so that we have $\Sigma f(-(r+1) / 2)=0$. If $r$ is even, $\Sigma f$ can be anti- $(r+1)$ reflective only if $\Sigma f(-r / 2)=-\Sigma f(-r / 2-1)=-(\Sigma f(-r / 2)-f(-r / 2))$; i.e., $\Sigma f(-r / 2)=\frac{1}{2} f(-r / 2)$.

Once we have found $x$ and $y$ such that $x+y+r+1=0$ and $\Sigma f(x)=$ $-\Sigma f(y)$, it is easy to see that we will also have $\Sigma f(x-1)=-\Sigma f(y+1)$, if $f$ is $r$-reflective, since $\Sigma f(x)-\Sigma f(x-1)=f(x)=f(y+1)=\Sigma f(y+1)-\Sigma f(y)$.

Suppose, on the other hand, that $f$ is anti-r-reflective. If $r$ is odd, clearly $\Sigma f(x)=\Sigma f(y)$ if $x=y=-(r+1) / 2$. If $r$ is even, then $f(-r / 2)=0$; so $\Sigma f(x)=\Sigma f(y)$ when $x=-r / 2$ and $y=-r / 2-1$. Once we have found $x$ and $y$ such that $x+y+r+1=0$ and $\Sigma f(x)=\Sigma f(y)$, it is easy to verify as above that $\Sigma f(x-1)=\Sigma f(y+1)$.

Lemma 3. If $f$ is any even function with $f(0)=0$, the $r$-fold repeated sum $\Sigma^{r} f$ is $r$-reflective for all even $r$ and anti-r-reflective for all odd $r$, if we choose the constant $C=0$ in each summation. If $f$ is any odd function, the r-fold repeated sum $\Sigma^{r} f$ is $r$-reflective for all odd $r$ and anti-r-reflective for all even $r$, if we choose the constant $C=0$ in each summation.

Proof. Note that $f(0)=0$ if $f$ is odd. If $f(0)=0$ and if we always choose $C=0$, it is easy to verify by induction on $r$ that $\Sigma^{r} f(x)=0$ for $-r \leq x \leq 0$. Therefore the choice $C=0$ always agrees with the unique choice stipulated in the proof of Lemma 2, whenever a specific value of $C$ is necessary in that lemma.

When $m$ is a positive integer, the function $f(x)=x^{m}$ obviously satisfies the condition of Lemma 3. Therefore we have proved that each function $\Sigma^{r} n^{m}$ is either $r$-reflective or anti-r-reflective, for all $r>0$ and $m>0$. And Faulhaber presumably knew this too. His theorem can now be proved if we supply one small additional fact, specializing from arbitrary functions to polynomials:

Lemma 4. A polynomial $f(x)$ is r-reflective if and only if it can be written as a polynomial in $x(x+r)$; it is anti-r-reflective if and only if it can be written as $(x+r / 2)$ times a polynomial in $x(x+r)$.

Proof. The second statement follows from the first, because we have already observed that an anti-r-reflective function must have $f(-r / 2)=0$ and because the function $x+r / 2$ is obviously anti-r-reflective. Furthermore, any polynomial in $x(x+r)$ is $r$-reflective, because $x(x+r)=y(y+r)$ when $x+y+r=0$. Conversely, if $f(x)$ is $r$-reflective, we have $f(x-r / 2)=f(-x-r / 2)$, so $g(x)=f(x-r / 2)$ is an even function of $x$; hence $g(x)=h\left(x^{2}\right)$ for some polynomial $h$. Then $f(x)=g(x+r / 2)=h\left(x(x+r)+r^{2} / 4\right)$ is a polynomial in $x(x+r)$.

Theorem (Faulhaber). There exist polynomials $g_{r, m}$ for all positive integers $r$ and $m$ such that

$$
\Sigma^{r} n^{2 m-1}=g_{r, 2 m+1}(n(n+r)) \Sigma^{r} n^{1}, \quad \Sigma^{r} n^{2 m}=g_{r, 2 m}(n(n+r)) \Sigma^{r} n^{2} .
$$

Proof. Lemma 3 tells us that $\Sigma^{r} n^{m}$ is $r$-reflective if $m+r$ is even and anti-rreflective if $m+r$ is odd. 
Note that $\Sigma^{r} n^{1}=\left(\begin{array}{c}n+r \\ r+1\end{array}\right)$. Therefore a polynomial in $n$ is a multiple of $\Sigma^{r} n^{1}$ if and only if it vanishes at $-r, \ldots,-1,0$. We have shown in the proof of Lemma 3 that $\Sigma^{r} n^{m}$ has this property for all $m$; therefore $\Sigma^{r} n^{m} / \Sigma^{r} n^{1}$ is an $r$-reflective polynomial when $m$ is odd, an anti-r-reflective polynomial when $m$ is even. In the former case, we are done, by Lemma 4. In the latter case, Lemma 4 establishes the existence of a polynomial $g$ such that $\Sigma^{r} n^{m} / \Sigma^{r} n^{1}=$ $(n+r / 2) g(n(n+r))$. Again, we are done, because the identity

$$
\Sigma^{r} n^{2}=\frac{2 n+r}{r+2} \Sigma^{r} n^{1}
$$

is readily verified.

\section{A Plausible Derivation}

Faulhaber probably did not think about $r$-reflective and anti-r-reflective functions in exactly the way we have described them, but his book [2] certainly indicates that he was quite familiar with the territory encompassed by that theory.

In fact, he could have found his formulas for power sums without knowing the theory in detail. A simple approach, illustrated here for $\Sigma n^{13}$, would suffice: Suppose

$$
14 \Sigma n^{13}=n^{7}(n+1)^{7}-S(n)
$$

where $S(n)$ is a 1-reflective function to be determined. Then

$$
\begin{aligned}
14 n^{13} & =n^{7}(n+1)^{7}-(n-1)^{7} n^{7}-\nabla S(n) \\
& =14 n^{13}+70 n^{11}+42 n^{9}+2 n^{7}-\nabla S(n),
\end{aligned}
$$

and we have

$$
S(n)=70 \Sigma n^{11}+42 n^{9}+2 \Sigma n^{7}
$$

In other words,

$$
\Sigma n^{13}=\frac{64}{7} N^{7}-5 \Sigma n^{11}-3 \Sigma n^{9}-\frac{1}{7} \Sigma n^{7}
$$

and we can complete the calculation by subtracting multiples of previously computed results.

The great advantage of using polynomials in $N$ rather than $n$ is that the new formulas are considerably shorter. The method Faulhaber and others had used before making this discovery was most likely equivalent to the laborious calculation

$$
\begin{aligned}
\Sigma n^{13}= & \frac{1}{14} n^{14}+\frac{13}{2} \Sigma n^{12}-26 \Sigma n^{11}+\frac{143}{2} \Sigma n^{10}-143 \Sigma n^{9}+\frac{429}{2} \Sigma n^{8}+\frac{1716}{7} \Sigma n^{7} \\
& +\frac{429}{2} \Sigma n^{6}-143 \Sigma n^{5}+\frac{143}{2} \Sigma n^{4}-26 \Sigma n^{3}+\frac{13}{2} \Sigma n^{2}-\Sigma n^{1}+\frac{1}{14} n
\end{aligned}
$$

the coefficients here are $\frac{1}{14}\left(\begin{array}{c}14 \\ 12\end{array}\right),-\frac{1}{14}\left(\begin{array}{c}14 \\ 11\end{array}\right), \ldots, \frac{1}{14}\left(\begin{array}{c}14 \\ 0\end{array}\right)$.

To handle sums of even exponents, Faulhaber knew that

$$
\Sigma n^{2 m}=\frac{n+\frac{1}{2}}{2 m+1}\left(a_{1} N+a_{2} N^{2}+\cdots+a_{m} N^{m}\right)
$$

holds if and only if

$$
\Sigma n^{2 m+1}=\frac{a_{1}}{2} N^{2}+\frac{a_{2}}{3} N^{3}+\cdots+\frac{a_{m}}{m+1} N^{m+1} .
$$


Therefore, he could get two sums for the price of one [2, folios C.iv verso and D.i recto]. It is not difficult to prove this relation by establishing an isomorphism between the calculations of $\Sigma n^{2 m+1}$ and the calculations of the quantities $S_{2 m}=\left((2 m+1) \Sigma n^{2 m}\right) /\left(n+\frac{1}{2}\right)$; for example, the recurrence for $\Sigma n^{13}$ above corresponds to the formula

$$
S_{12}=64 N^{6}-5 S_{10}-3 S_{8}-\frac{1}{7} S_{6},
$$

which can be derived in essentially the same way. Since the recurrences are essentially identical, we obtain a correct formula for $\Sigma n^{2 m+1}$ from the formula for $S_{2 m}$ if we replace $N^{k}$ everywhere by $N^{k+1} /(k+1)$.

\section{FAULHABER'S CRYPTOMATH}

Mathematicians of Faulhaber's day tended to conceal their methods and hide results in secret code. Faulhaber ends his book [2] with a curious exercise of this kind, evidently intended to prove to posterity that he had in fact computed the formulas for sums of powers as far as $\Sigma n^{25}$ although he published the results only up to $\Sigma n^{17}$.

His puzzle can be translated into modern notation as follows. Let

$$
\Sigma^{9} n^{8}=\frac{a_{17} n^{17}+\cdots+a_{2} n^{2}+a_{1} n}{d}
$$

where the $a$ 's are integers having no common factor and $d=a_{17}+\cdots+a_{2}+a_{1}$. Let

$$
\Sigma n^{25}=\frac{A_{26} n^{26}+\cdots+A_{2} n^{2}+A_{1} n}{D}
$$

be the analogous formula for $\Sigma n^{25}$. Let

$$
\begin{aligned}
& \Sigma n^{22}=\frac{\left(b_{10} N^{10}-b_{9} N^{9}+\cdots+b_{0}\right)}{b_{10}-b_{9}+\cdots+b_{0}} \Sigma n^{2}, \\
& \Sigma n^{23}=\frac{\left(c_{10} n^{10}-c_{9} N^{9}+\cdots+c_{0}\right)}{c_{10}-c_{9}+\cdots+c_{0}} \Sigma n^{3}, \\
& \Sigma n^{24}=\frac{\left(d_{11} n^{11}-d_{10} N^{10}+\cdots-d_{0}\right)}{d_{11}-d_{10}+\cdots-d_{0}} \Sigma n^{2}, \\
& \Sigma n^{25}=\frac{\left(e_{11} n^{11}-e_{10} N^{10}+\cdots-e_{0}\right)}{e_{11}-e_{10}+\cdots-e_{0}} \Sigma n^{3},
\end{aligned}
$$

where the integers $b_{k}, c_{k}, d_{k}, e_{k}$ are as small as possible so that $b_{k}, c_{k}, d_{k}, e_{k}$ are multiples of $2^{k}$. (He wants them to be multiples of $2^{k}$ so that $b_{k} N^{k}, c_{k} N^{k}$, $d_{k} N^{k}, e_{k} N^{k}$ are polynomials in $n$ with integer coefficients; that is why he wrote, for example, $\Sigma n^{7}=\left(12 N^{2}-8 N+2\right) N^{2} / 6$ instead of $\left(6 N^{2}-4 N+1\right) N^{2} / 3$. See [2, folio D.i verso].) Then compute

$$
\begin{aligned}
& x_{1}=\left(c_{3}-a_{12}\right) / 7924252 ; \\
& x_{2}=\left(b_{5}+a_{10}\right) / 112499648 ; \\
& x_{3}=\left(a_{11}-b_{9}-c_{1}\right) / 2945002 ; \\
& x_{4}=\left(a_{14}+c_{7}\right) / 120964 ; \\
& x_{5}=\left(A_{26} a_{11}-D+a_{13}+d_{11}+e_{11}\right) / 199444 .
\end{aligned}
$$


These values $\left(x_{1}, x_{2}, x_{3}, x_{4}, x_{5}\right)$ specify the five letters of a what he called a "hochgerühmte Nam," if we use five designated alphabets [2, folio F.i recto].

It is doubtful whether anybody solved this puzzle during the first 360 years after its publication, but the task is relatively easy with modern computers. We have

$$
\begin{gathered}
a_{10}=532797408, \quad a_{11}=104421616, \quad a_{12}=14869764, \\
a_{13}=1526532, \quad a_{14}=110160 ; \\
b_{5}=29700832, \quad b_{9}=140800 ; \\
c_{1}=205083120, \quad c_{3}=344752128, \quad c_{7}=9236480 ; \\
d_{11}=559104 ; \quad e_{11}=86016 ; \quad A_{26}=42 ; \quad D=1092 .
\end{gathered}
$$

The fact that $x_{2}=(29700832+532797408) / 112499648=5$ is an integer is reassuring: We must be on the right track! But alas, the other values are not integral.

A bit of experimentation soon reveals that we do obtain good results if we divide all the $c_{k}$ by 4 . Then, for example,

$$
x_{1}=(344752128 / 4-14869764) / 7924252=9,
$$

and we also find $x_{3}=18, x_{4}=20$. It appears that Faulhaber calculated $\Sigma^{9} n^{8}$ and $\Sigma n^{22}$ correctly, and that he also had a correct expression for $\Sigma n^{23}$ as a polynomial in $N$; but he probably never went on to express $\Sigma n^{23}$ as a polynomial in $n$, because he would then have multiplied his coefficients by 4 in order to compute $c_{6} N^{6}$ with integer coefficients.

The values of $\left(x_{1}, x_{2}, x_{3}, x_{4}\right)$ correspond to the letters I E S U, so the concealed name in Faulhaber's riddle is undoubtedly I E S U S (Jesus).

But his formula for $x_{5}$ does not check out at all; it is way out of range and not an integer. This is the only formula that relates to $\Sigma n^{24}$ and $\Sigma n^{25}$, and it involves only the simplest elements of those sums-the leading coefficients $A_{26}$, $D, d_{11}, e_{11}$. Therefore, we have no evidence that Faulhaber's calculations beyond $\Sigma n^{23}$ were reliable. It is tempting to imagine that he meant to say ' $A_{26} a_{11} / D$ ' instead of ' $A_{26} a_{11}-D$ ' in his formula for $x_{5}$, but even then major corrections are needed to the other terms and it is unclear what he intended.

\section{AlL-INTEGER FORMULAS}

Faulhaber's theorem allows us to express the power sum $\Sigma n^{m}$ in terms of about $\frac{1}{2} m$ coefficients. The elementary theory above also suggests another approach that produces a similar effect: We can write, for example,

$$
\begin{aligned}
n & =\left(\begin{array}{c}
n \\
1
\end{array}\right) ; \\
n^{3} & =6\left(\begin{array}{c}
n+1 \\
3
\end{array}\right)+\left(\begin{array}{c}
n \\
1
\end{array}\right) ; \\
n^{5} & =120\left(\begin{array}{c}
n+2 \\
5
\end{array}\right)+30\left(\begin{array}{c}
n+1 \\
3
\end{array}\right)+\left(\begin{array}{c}
n \\
1
\end{array}\right) .
\end{aligned}
$$

(It is easy to see that any odd function $g(n)$ of the integer $n$ can be expressed uniquely as a linear combination

$$
g(n)=a_{1}\left(\begin{array}{l}
n \\
1
\end{array}\right)+a_{3}\left(\begin{array}{c}
n+1 \\
3
\end{array}\right)+a_{5}\left(\begin{array}{c}
n+2 \\
5
\end{array}\right)+\cdots
$$

of the odd functions $\left(\begin{array}{l}n \\ 1\end{array}\right),\left(\begin{array}{c}n+1 \\ 3\end{array}\right),\left(\begin{array}{c}n+2 \\ 5\end{array}\right), \ldots$, because we can determine the coefficients $a_{1}, a_{3}, a_{5}, \ldots$ successively by plugging in the values $n=1,2$, 
$3, \ldots$. The coefficients $a_{k}$ will be integers if and only if $g(n)$ is an integer for all $n$.) Once $g(n)$ has been expressed in this way, we clearly have

$$
\Sigma g(n)=a_{1}\left(\begin{array}{c}
n+1 \\
2
\end{array}\right)+a_{3}\left(\begin{array}{c}
n+2 \\
4
\end{array}\right)+a_{5}\left(\begin{array}{c}
n+3 \\
6
\end{array}\right)+\cdots .
$$

This approach, therefore, yields the following identities for sums of odd powers:

$$
\begin{aligned}
\Sigma n^{1}= & \left(\begin{array}{c}
n+1 \\
2
\end{array}\right) ; \\
\Sigma n^{3}= & 6\left(\begin{array}{c}
n+2 \\
4
\end{array}\right)+\left(\begin{array}{c}
n+1 \\
2
\end{array}\right) ; \\
\Sigma n^{5}= & 120\left(\begin{array}{c}
n+3 \\
6
\end{array}\right)+30\left(\begin{array}{c}
n+2 \\
4
\end{array}\right)+\left(\begin{array}{c}
n+1 \\
2
\end{array}\right) ; \\
\Sigma n^{7}= & 5040\left(\begin{array}{c}
n+4 \\
8
\end{array}\right)+1680\left(\begin{array}{c}
n+3 \\
6
\end{array}\right)+126\left(\begin{array}{c}
n+2 \\
4
\end{array}\right)+\left(\begin{array}{c}
n+1 \\
2
\end{array}\right) ; \\
\Sigma n^{9}= & 362880\left(\begin{array}{c}
n+5 \\
10
\end{array}\right)+151200\left(\begin{array}{c}
n+4 \\
8
\end{array}\right)+17640\left(\begin{array}{c}
n+3 \\
6
\end{array}\right) \\
& +510\left(\begin{array}{c}
n+2 \\
4
\end{array}\right)+\left(\begin{array}{c}
n+1 \\
2
\end{array}\right) ; \\
\Sigma n^{11}= & 39916800\left(\begin{array}{c}
n+6 \\
12
\end{array}\right)+19958400\left(\begin{array}{c}
n+5 \\
10
\end{array}\right)+3160080\left(\begin{array}{c}
n+4 \\
8
\end{array}\right) \\
& +168960\left(\begin{array}{c}
n+3 \\
6
\end{array}\right)+2046\left(\begin{array}{c}
n+2 \\
4
\end{array}\right)+\left(\begin{array}{c}
n+1 \\
2
\end{array}\right) ; \\
\Sigma n^{13}= & 6227020800\left(\begin{array}{c}
n+7 \\
14
\end{array}\right)+3632428800\left(\begin{array}{c}
n+6 \\
12
\end{array}\right)+726485760\left(\begin{array}{c}
n+5 \\
10
\end{array}\right) \\
& +57657600\left(\begin{array}{c}
n+4 \\
8
\end{array}\right)+1561560\left(\begin{array}{c}
n+3 \\
6
\end{array}\right)+8190\left(\begin{array}{c}
n+2 \\
4
\end{array}\right)+\left(\begin{array}{c}
n+1 \\
2
\end{array}\right) .
\end{aligned}
$$

And repeated sums are equally easy; we have

$$
\Sigma^{r} n^{1}=\left(\begin{array}{c}
n+r \\
1+r
\end{array}\right), \quad \Sigma^{r} n^{3}=6\left(\begin{array}{c}
n+1+r \\
3+r
\end{array}\right)+\left(\begin{array}{c}
n+r \\
1+r
\end{array}\right), \quad \text { etc. }
$$

The coefficients in these formulas are related to what Riordan [7, p. 213] has called central factorial numbers of the second kind. In his notation

$$
x^{m}=\sum_{k=1}^{m} T(m, k) x^{[k]}, \quad x^{[k]}=x\left(x+\frac{k}{2}-1\right)\left(x+\frac{k}{2}-2\right) \cdots\left(x-\frac{k}{2}+1\right),
$$

when $m>0$, and $T(m, k)=0$ when $m-k$ is odd; hence

$$
\begin{aligned}
n^{2 m-1} & =\sum_{k=1}^{m}(2 k-1) ! T(2 m, 2 k)\left(\begin{array}{c}
n+k-1 \\
2 k-1
\end{array}\right), \\
\Sigma n^{2 m-1} & =\sum_{k=1}^{m}(2 k-1) ! T(2 m, 2 k)\left(\begin{array}{c}
n+k \\
2 k
\end{array}\right) .
\end{aligned}
$$

The coefficients $T(2 m, 2 k)$ are always integers, because the basic identity $x^{[k+2]}=x^{[k]}\left(x^{2}-k^{2} / 4\right)$ implies the recurrence

$$
T(2 m+2,2 k)=k^{2} T(2 m, 2 k)+T(2 m, 2 k-2) .
$$


The generating function for these numbers turns out to be

$$
\cosh (2 x \sinh (y / 2))=\sum_{m=0}^{\infty}\left(\sum_{k=0}^{m} T(2 m, 2 k) x^{2 k}\right) \frac{y^{2 m}}{(2 m) !} .
$$

Notice that the power-sum formulas obtained in this way are more "efficient" than the well-known formulas based on Stirling numbers (see [5, (6.12)]):

$$
\Sigma n^{m}=\sum_{k} k !\left\{\begin{array}{l}
m \\
k
\end{array}\right\}\left(\begin{array}{l}
n+1 \\
k+1
\end{array}\right)=\sum_{k} k !\left\{\begin{array}{l}
m \\
k
\end{array}\right\}(-1)^{m-k}\left(\begin{array}{l}
n+k \\
k+1
\end{array}\right) .
$$

The latter formulas give, for example,

$$
\begin{aligned}
\Sigma n^{7}= & 5040\left(\begin{array}{c}
n+1 \\
8
\end{array}\right)+15120\left(\begin{array}{c}
n+1 \\
7
\end{array}\right)+16800\left(\begin{array}{c}
n+1 \\
6
\end{array}\right)+8400\left(\begin{array}{c}
n+1 \\
5
\end{array}\right)+1806\left(\begin{array}{c}
n+1 \\
4
\end{array}\right) \\
& +126\left(\begin{array}{c}
n+1 \\
3
\end{array}\right)+\left(\begin{array}{c}
n+1 \\
2
\end{array}\right) \\
= & 5040\left(\begin{array}{c}
n+7 \\
8
\end{array}\right)-15120\left(\begin{array}{c}
n+6 \\
7
\end{array}\right)+16800\left(\begin{array}{c}
n+5 \\
6
\end{array}\right)-8400\left(\begin{array}{c}
n+4 \\
5
\end{array}\right)+1806\left(\begin{array}{c}
n+3 \\
4
\end{array}\right) \\
& -126\left(\begin{array}{c}
n+2 \\
3
\end{array}\right)+\left(\begin{array}{c}
n+1 \\
2
\end{array}\right) .
\end{aligned}
$$

There are about twice as many terms, and the coefficients are larger. (The Faulhaberian expression $\Sigma n^{7}=\left(6 N^{4}-4 N^{3}+N^{2}\right) / 3$ is, of course, better yet.)

Similar formulas for even powers can be obtained as follows. We have

$$
\begin{aligned}
n^{2}=n\left(\begin{array}{c}
n \\
1
\end{array}\right) & =U_{1}(n), \\
n^{4}=6 n\left(\begin{array}{c}
n+1 \\
3
\end{array}\right)+n\left(\begin{array}{c}
n \\
1
\end{array}\right) & =12 U_{2}(n)+U_{1}(n), \\
n^{6}=120 n\left(\begin{array}{c}
n+2 \\
5
\end{array}\right)+30 n\left(\begin{array}{c}
n+1 \\
3
\end{array}\right)+n\left(\begin{array}{l}
n \\
1
\end{array}\right) & =360 U_{3}(n)+60 U_{2}(n)+U_{1}(n),
\end{aligned}
$$

etc., where

$$
U_{k}(n)=\frac{n}{k}\left(\begin{array}{c}
n+k-1 \\
2 k-1
\end{array}\right)=\left(\begin{array}{c}
n+k \\
2 k
\end{array}\right)+\left(\begin{array}{c}
n+k-1 \\
2 k
\end{array}\right) .
$$

Hence

$$
\begin{aligned}
\Sigma n^{2}= & T_{1}(n) \\
\Sigma n^{4}= & 12 T_{2}(n)+T_{1}(n) \\
\Sigma n^{6}= & 360 T_{3}(n)+60 T_{2}+T_{1}(n) \\
\Sigma n^{8}= & 20160 T_{4}(n)+5040 T_{3}(n)+252 T_{2}(n)+T_{1}(n) \\
\Sigma n^{10}= & 1814400 T_{5}(n)+604800 T_{4}(n)+52920 T_{3}(n)+1020 T_{2}(n)+T_{1}(n) \\
\Sigma n^{12}= & 239500800 T_{6}(n)+99792000 T_{5}(n)+12640320 T_{4}(n) \\
& +506880 T_{3}(n)+4092 T_{2}(n)+T_{1}(n)
\end{aligned}
$$

etc., where

$$
T_{k}(n)=\left(\begin{array}{c}
n+k+1 \\
2 k+1
\end{array}\right)+\left(\begin{array}{c}
n+k \\
2 k+1
\end{array}\right)=\frac{2 n+1}{2 k+1}\left(\begin{array}{c}
n+k \\
2 k
\end{array}\right) .
$$

Curiously, we have found a relation here between $\Sigma n^{2 m}$ and $\Sigma n^{2 m-1}$, somewhat analogous to Faulhaber's relation between $\Sigma n^{2 m}$ and $\Sigma n^{2 m+1}$ : The formula

$$
\frac{\Sigma n^{2 m}}{2 n+1}=a_{1}\left(\begin{array}{c}
n+1 \\
2
\end{array}\right)+a_{2}\left(\begin{array}{c}
n+2 \\
4
\end{array}\right)+\cdots+a_{m}\left(\begin{array}{c}
n+m \\
2 m
\end{array}\right)
$$


holds if and only if

$$
\Sigma n^{2 m-1}=\frac{3}{1} a_{1}\left(\begin{array}{c}
n+1 \\
2
\end{array}\right)+\frac{5}{2} a_{2}\left(\begin{array}{c}
n+2 \\
4
\end{array}\right)+\cdots+\frac{2 m+1}{m} a_{m}\left(\begin{array}{c}
n+m \\
2 m
\end{array}\right) .
$$

\section{REFLECTIVE DECOMPOSITION}

The forms of the expressions in the previous section lead naturally to useful representations of arbitrary functions $f(n)$ defined on the integers. It is easy to see that any $f(n)$ can be written uniquely in the form

$$
f(n)=\sum_{k \geq 0} a_{k}\left(\begin{array}{c}
n+\lfloor k / 2\rfloor \\
k
\end{array}\right)
$$

for some coefficients $a_{k}$; indeed, we have

$$
a_{k}=\nabla^{k} f(\lfloor k / 2\rfloor) \text {. }
$$

(Thus $a_{0}=f(0), a_{1}=f(0)-f(-1), a_{2}=f(1)-2 f(0)+f(-1)$, etc.) The $a_{k}$ are integers if and only if $f(n)$ is always an integer. The $a_{k}$ are eventually zero if and only if $f$ is a polynomial. The $a_{2 k}$ are all zero if and only if $f$ is odd. The $a_{2 k+1}$ are all zero if and only if $f$ is 1 -reflective.

Similarly, there is a unique expansion

$$
f(n)=b_{0} T_{0}(n)+b_{1} U_{1}(n)+b_{2} T_{1}(n)+b_{3} U_{2}(n)+b_{4} T_{2}(n)+\cdots,
$$

in which the $b_{k}$ are integers if and only if $f(n)$ is always an integer. The $b_{2 k}$ are all zero if and only if $f$ is even and $f(0)=0$. The $b_{2 k+1}$ are all zero if and only if $f$ is anti-1-reflective. Using the recurrence relations

$$
\nabla T_{k}(n)=U_{k}(n), \quad \nabla U_{k}(n)=T_{k-1}(n-1),
$$

we find

and therefore

$$
a_{k}=\nabla^{k} f(\lfloor k / 2\rfloor)=2 b_{k-1}+(-1)^{k} b_{k}
$$

$$
b_{k}=\sum_{j=0}^{k}(-1)^{\lceil j / 2\rceil+\lfloor k / 2\rfloor} 2^{k-j} a_{j} .
$$

In particular, when $f(n)=1$ for all $n$, we have $b_{k}=(-1)^{\lfloor k / 2\rfloor} 2^{k}$. The infinite series is finite for each $n$.

Theorem. If $f$ is any function defined on the integers and if $r, s$ are arbitrary integers, we can always express $f$ in the form

$$
f(n)=g(n)+h(n)
$$

where $g(n)$ is $r$-reflective and $h(n)$ is anti-s-reflective. This representation is unique, except when $r$ is even and $s$ is odd; in the latter case the representation is unique if we specify the value of $g$ or $h$ at any point.

Proof. It suffices to consider $0 \leq r, s \leq 1$, because $f(x)$ is (anti)-r-reflective if and only if $f(x+a)$ is (anti)- $(r+2 a)$-reflective.

When $r=s=0$, the result is just the well-known decomposition of a function into even and odd parts,

$$
g(n)=\frac{1}{2}(f(n)+f(-n)), \quad h(n)=\frac{1}{2}(f(n)-f(-n)) .
$$


When $r=s=1$, we have similarly

$$
g(n)=\frac{1}{2}(f(n)+f(-1-n)), \quad h(n)=\frac{1}{2}(f(n)-f(-1-n)) .
$$

When $r=1$ and $s=0$, it is easy to deduce that $h(0)=0, g(0)=f(0)$, $h(1)=f(0)-f(-1), g(1)=f(1)-f(0)+f(-1), h(2)=f(1)-f(0)+f(-1)-$ $f(-2), g(2)=f(2)-f(1)+f(0)-f(-1)+f(-2)$, etc.

And when $r=0$ and $s=1$, the general solution is $g(0)=f(0)-C$, $h(0)=C, g(1)=f(-1)+C, h(1)=f(1)-f(-1)-C, g(2)=f(1)-$ $f(-1)+f(-2)-C, h(2)=f(2)-f(1)+f(-1)-f(-2)+C$, etc.

When $f(n)=\sum_{k \geq 0} a_{k}\left(\begin{array}{c}n+\lfloor k / 2\rfloor \\ k\end{array}\right)$, the case $r=1$ and $s=0$ corresponds to the decomposition

$$
g(n)=\sum_{k=0}^{\infty} a_{2 k}\left(\begin{array}{c}
n+k \\
2 k
\end{array}\right), \quad h(n)=\sum_{k=0}^{\infty} a_{2 k+1}\left(\begin{array}{c}
n+k \\
2 k+1
\end{array}\right) .
$$

Similarly, the representation $f(n)=\sum_{k \geq 0} b_{2 k} T_{k}(n)+\sum_{k \geq 0} b_{2 k+1} U_{k+1}(n)$ corresponds to the case $r=0, s=1, C=f(0)$.

\section{BACK TO FAULHABER'S FORM}

Let us now return to representations of $\Sigma n^{m}$ as polynomials in $n(n+1)$. Setting $u=2 N=n^{2}+n$, we have

$$
\begin{array}{rlrl}
\Sigma n & =\frac{1}{2} u & & =\frac{1}{2} A_{0}^{(1)} u, \\
\Sigma n^{3} & =\frac{1}{4} u^{2} & & \frac{1}{4}\left(A_{0}^{(2)} u^{2}+A_{1}^{(1)} u\right), \\
\Sigma n^{5} & =\frac{1}{6}\left(u^{3}-\frac{1}{2} u^{2}\right) & & \frac{1}{6}\left(A_{0}^{(3)} u^{3}+A_{1}^{(3)} u^{2}+A_{2}^{(3)} u\right), \\
\Sigma n^{7} & =\frac{1}{8}\left(u^{4}-\frac{4}{3} u^{3}+\frac{2}{3} u^{2}\right) & =\frac{1}{8}\left(A_{0}^{(4)} u^{4}+A_{1}^{(4)} u^{3}+A_{2}^{(4)} u^{2}+A_{3}^{(4)} u\right),
\end{array}
$$

and so on, for certain coefficients $A_{k}^{(m)}$.

Faulhaber never discovered the Bernoulli numbers; i.e., he never realized that a single sequence of constants $B_{0}, B_{1}, B_{2}, \ldots$ would provide a uniform formula

$$
\Sigma n^{m}=\frac{1}{m+1}\left(B_{0} n^{m+1}-\left(\begin{array}{c}
m+1 \\
1
\end{array}\right) B_{1} n^{m}+\left(\begin{array}{c}
m+1 \\
2
\end{array}\right) B_{2} n^{m-1}-\cdots+(-1)^{m}\left(\begin{array}{c}
m+1 \\
m
\end{array}\right) B_{m} n\right)
$$

for all sums of powers. He never mentioned, for example, the fact that almost half of the coefficients turned out to be zero after he had converted his formulas for $\Sigma n^{m}$ from polynomials in $N$ to polynomials in $n$. (He did notice that the coefficient of $n$ was zero when $m>1$ was odd.)

However, we know now that Bernoulli numbers exist, and we know that $B_{3}=$ $B_{5}=B_{7}=\cdots=0$. This is a strong condition. Indeed, it completely defines the constants $A_{k}^{(m)}$ in the Faulhaber polynomials above, given that $A_{0}^{(m)}=1$.

For example, let us consider the case $m=4$, i.e., the formula for $\Sigma n^{7}$ : We need to find coefficients $a=A_{1}^{(4)}, b=A_{2}^{(4)}, c=A_{3}^{(4)}$ such that the polynomial

$$
n^{4}(n+1)^{4}+a n^{3}(n+1)^{3}+b n^{2}(n+1)^{2}+c n(n+1)
$$


has vanishing coefficients of $n^{5}, n^{3}$, and $n$. The polynomial is

$$
\begin{aligned}
n^{8}+4 n^{7}+6 n^{6}+4 n^{5} & +n^{4} \\
+a n^{6}+3 a n^{5} & +3 a n^{4}+a n^{3} \\
& +b n^{4}+2 b n^{3}+n^{2} \\
& +c n^{2}+c n
\end{aligned}
$$

so we must have $3 a+4=2 b+a=c=0$. In general the coefficient of, say, $n^{2 m-5}$ in the polynomial for $2 m \Sigma n^{2 m-1}$ is easily seen to be

$$
\left(\begin{array}{c}
m \\
5
\end{array}\right) A_{0}^{(m)}+\left(\begin{array}{c}
m-1 \\
3
\end{array}\right) A_{1}^{(m)}+\left(\begin{array}{c}
m-2 \\
1
\end{array}\right) A_{2}^{(m)} .
$$

Thus, the Faulhaber coefficients can be defined by the rules

$$
A_{0}^{(w)}=1 ; \quad \sum_{j=0}^{k}\left(\begin{array}{c}
w-j \\
2 k+1-2 j
\end{array}\right) A_{j}^{(w)}=0, \quad k>0 .
$$

(The upper parameter will often be called $w$ instead of $m$, in the sequel, because we will want to generalize to noninteger values.) Notice that (*) defines the coefficients for each exponent without reference to other exponents; for every integer $k \geq 0$, the quantity $A_{k}^{(w)}$ is a certain rational function of $w$. For example, we have

$$
\begin{aligned}
-A_{1}^{(w)}= & w(w-2) / 6 \\
A_{2}^{(w)}= & w(w-1)(w-3)(7 w-8) / 360 \\
-A_{3}^{(w)}= & w(w-1)(w-2)(w-4)\left(31 w^{2}-89 w+48\right) / 15120 \\
A_{4}^{(w)}= & w(w-1)(w-2)(w-3)(w-5) \\
& \cdot\left(127 w^{3}-691 w^{2}+1038 w-384\right) / 6048000
\end{aligned}
$$

and in general $A_{k}^{(w)}$ is $w^{\underline{k}}=w(w-1) \cdots(w-k+1)$ times a polynomial of degree $k$, with leading coefficient equal to $\left(2-2^{2 k}\right) B_{2 k} /(2 k)$ !; if $k>0$, that polynomial vanishes when $w=k+1$.

Jacobi mentioned these coefficients $A_{k}^{(m)}$ in his paper [6], and tabulated them for $m \leq 6$, although he did not consider the recurrence $(*)$. He observed that the derivative of $\Sigma n^{m}$ with respect to $n$ is $m \Sigma n^{m-1}+B_{m}$; this follows because power sums can be expressed in terms of Bernoulli polynomials,

$$
\Sigma n^{m}=\frac{1}{m+1}\left(B_{m+1}(n+1)-B_{m+1}(0)\right),
$$

and because $B_{m}^{\prime}(x)=m B_{m-1}(x)$. Thus Jacobi obtained a new proof of Faulhaber's formulas for even exponents:

$$
\begin{aligned}
& \Sigma n^{2}=\frac{1}{3}\left(\frac{2}{4} A_{0}^{(2)} u+\frac{1}{4} A_{1}^{(2)}\right)(2 n+1), \\
& \Sigma n^{4}=\frac{1}{5}\left(\frac{3}{6} A_{0}^{(3)} u^{2}+\frac{2}{6} A_{1}^{(3)} u+\frac{1}{6} A_{2}^{(3)}\right)(2 n+1), \\
& \Sigma n^{6}=\frac{1}{7}\left(\frac{4}{8} A_{0}^{(4)} u^{3}+\frac{3}{8} A_{1}^{(4)} u^{2}+\frac{2}{8} A_{2}^{(4)} u+\frac{1}{8} A_{3}^{(4)}\right)(2 n+1),
\end{aligned}
$$

etc. (The constant terms are zero, but they are shown explicitly here so that the 
pattern is plain.) Differentiating again gives, e.g.,

$$
\begin{aligned}
& \Sigma n^{5}=\frac{1}{6 \cdot 7 \cdot 8}\left(\left(4 \cdot 3 A_{0}^{(4)} u^{2}+3 \cdot 2 A_{1}^{(4)} u+2 \cdot 1 A_{2}^{(4)}\right)(2 n+1)^{2}\right. \\
&\left.\quad+2\left(4 A_{0}^{(4)} u^{3}+3 A_{1}^{(4)} u^{2}+2 A_{2}^{(4)} u+1 A_{3}^{(4)}\right)\right)-\frac{1}{6} B_{6} \\
&=\frac{1}{6 \cdot 7 \cdot 8}\left(8 \cdot 7 A_{0}^{(4)} u^{3}+\left(6 \cdot 5 A_{1}^{(4)}+4 \cdot 3 A_{0}^{(4)}\right) u^{2}\right. \\
&\left.\quad+\left(4 \cdot 3 A_{2}^{(4)}+3 \cdot 2 A_{1}^{(4)}\right) u+\left(2 \cdot 1 A_{3}^{(4)}+2 \cdot 1 A_{2}^{(4)}\right)\right)-\frac{1}{6} B_{6} .
\end{aligned}
$$

This yields Jacobi's recurrence

(**) $(2 w-2 k)(2 w-2 k-1) A_{k}^{(w)}+(w-k+1)(w-k) A_{k-1}^{(w)}=2 w(2 w-1) A_{k}^{(w-1)}$,

which is valid for all integers $w>k+1$, so it must be valid for all $w$. Our derivation of $(* *)$ also allows us to conclude that

$$
A_{m-2}^{(m)}=\left(\begin{array}{c}
2 m \\
2
\end{array}\right) B_{2 m-2}, \quad m \geq 2
$$

by considering the constant term of the second derivative of $\Sigma n^{2 m-1}$.

Recurrence $(*)$ does not define $A_{m}^{(m)}$, except as the limit of $A_{m}^{(w)}$ when $w \rightarrow m$. But we can compute this value by setting $w=m+1$ and $k=m$ in $(* *)$, which reduces to

$$
2 A_{m-1}^{(m+1)}=(2 m+2)(2 m+1) A_{m}^{(m)}
$$

because $A_{m}^{(m+1)}=0$. Thus,

$$
A_{m}^{(m)}=B_{2 m}, \quad \text { integer } m \geq 0 .
$$

\section{SOlution to THE RECURRENCE}

An explicit formula for $A_{k}^{(m)}$ can be found as follows: We have

$$
\Sigma n^{2 m-1}=\frac{1}{2 m}\left(B_{2 m}(n+1)-B_{2 m}\right)=\frac{1}{2 m}\left(A_{0}^{(m)} u^{m}+\cdots+A_{m-1}^{(m)} u\right),
$$

and $n+1=(\sqrt{1+4 u}+1) / 2$; hence, using the known values of $A_{m}^{(m)}$, we obtain

$$
\sum_{k=0}^{\infty} A_{k}^{(m)} u^{m-k}=B_{2 m}\left(\frac{\sqrt{1+4 u}+1}{2}\right)=B_{2 m}\left(\frac{1-\sqrt{1+4 u}}{2}\right),
$$

a closed form in terms of Bernoulli polynomials. (We have used the fact that $A_{m+1}^{(m)}=A_{m+2}^{(m)}=\cdots=0$, together with the identity $\left.B_{n}(x+1)=(-1)^{n} B_{n}(-x).\right)$ Expanding the right side in powers of $u$ gives

$$
\begin{aligned}
\sum_{l}\left(\begin{array}{c}
2 m \\
l
\end{array}\right) & \left(\frac{1-\sqrt{1+4 u}}{2}\right)^{l} B_{2 m-l} \\
= & \sum_{j, l}\left(\begin{array}{c}
2 m \\
l
\end{array}\right)\left(\begin{array}{c}
2 j+l \\
j
\end{array}\right) \frac{l}{2 j+l}(-u)^{j+l} B_{2 m-l},
\end{aligned}
$$

using equation (5.70) of [5]. Setting $j+l=m-k$ finally yields $A_{k}^{(m)}=(-1)^{m-k} \sum_{j}\left(\begin{array}{c}2 m \\ m-k-j\end{array}\right)\left(\begin{array}{c}m-k+j \\ j\end{array}\right) \frac{m-k-j}{m-k+j} B_{m+k+j}, \quad 0 \leq k<m$. 
This formula, which was first obtained by Gessel and Viennot [4], makes it easy to confirm that $A_{m-1}^{(m)}=0$ and $A_{m-2}^{(m)}=\left(\begin{array}{c}2 m \\ 2\end{array}\right) B_{2 m-2}$, and to derive additional values such as

$$
\begin{aligned}
& A_{m-3}^{(m)}=-2\left(\begin{array}{c}
2 m \\
2
\end{array}\right) B_{2 m-2}=-2 A_{m-2}^{(m)}, \quad m \geq 3 ; \\
& A_{m-4}^{(m)}=\left(\begin{array}{c}
2 m \\
4
\end{array}\right) B_{2 m-4}+5\left(\begin{array}{c}
2 m \\
2
\end{array}\right) B_{2 m-2}, \quad m \geq 4 .
\end{aligned}
$$

The author's interest in Faulhaber polynomials was inspired by the work of Edwards [1], who resurrected Faulhaber's work after it had been long forgotten and undervalued by historians of mathematics. Ira Gessel responded to the same stimulus by submitting problem E3204 to the Math Monthly [3] regarding a bivariate generating function for Faulhaber's coefficients. Such a function is obtainable from the univariate generating function above, using the standard generating function for Bernoulli polynomials: Since

$$
\begin{aligned}
\sum B_{2 m}\left(\frac{x+1}{2}\right) \frac{z^{2 m}}{(2 m) !} & =\frac{1}{2} \sum B_{m}\left(\frac{x+1}{2}\right) \frac{z^{m}}{m !}+\frac{1}{2} \sum B_{m}\left(\frac{x+1}{2}\right) \frac{(-z)^{m}}{m !} \\
& =\frac{z e^{(x+1) z / 2}}{2\left(e^{z}-1\right)}-\frac{z e^{-(x+1) z / 2}}{2\left(e^{-z}-1\right)}=\frac{z \cosh (x z / 2)}{2 \sinh (z / 2)},
\end{aligned}
$$

we have

$$
\begin{aligned}
\sum_{k, m} A_{k}^{(m)} u^{m-k} \frac{z^{2 m}}{(2 m) !} & =\sum_{m} B_{2 m}\left(\frac{\sqrt{1+4 u}+1}{2}\right) \frac{z^{2 m}}{(2 m) !} \\
& =\frac{z}{2} \frac{\cosh (\sqrt{1+4 u} z / 2)}{\sinh (z / 2)} ; \\
\sum_{k, m} A_{k}^{(m)} u^{k} \frac{z^{2 m}}{(2 m) !} & =\frac{z \sqrt{u} \cosh (\sqrt{u+4} z / 2)}{2 \sinh (z \sqrt{u} / 2)} .
\end{aligned}
$$

The numbers $A_{k}^{(m)}$ are obtainable by inverting a lower triangular matrix, as Edwards showed; indeed, recurrence $(*)$ defines such a matrix. Gessel and Viennot [4] observed that we can therefore express them in terms of a $k \times k$ determinant,

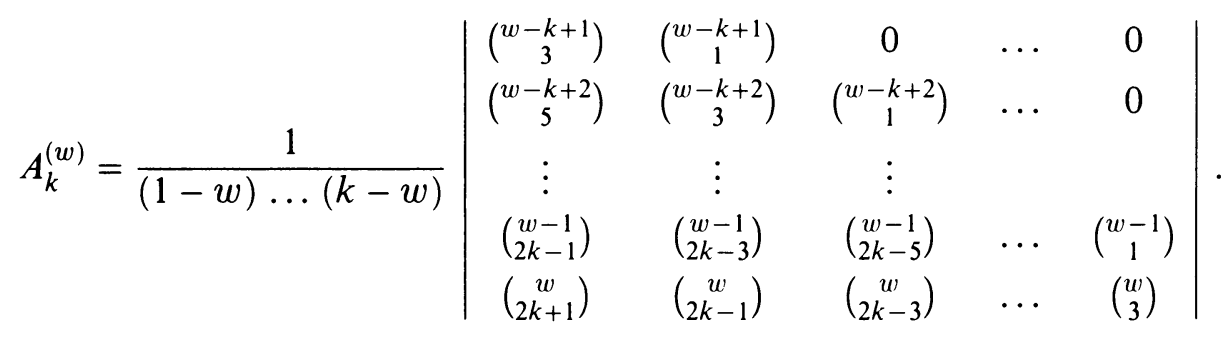

When $w$ and $k$ are positive integers, Gessel and Viennot proved that this determinant is the number of sequences of positive integers $a_{1} a_{2} a_{3} \cdots a_{3 k}$ such that

$$
\begin{gathered}
a_{3 j-2}<a_{3 j-1}<a_{3 j} \leq w-k+j \quad \text { for } 1 \leq j \leq k, \\
a_{3 j-2}<a_{3 j+1}, \quad a_{3 j-1}<a_{3 j+3} \text { for } 1 \leq j<k .
\end{gathered}
$$


In other words, it is the number of ways to put positive integers into a $k$-rowed triple staircase such as

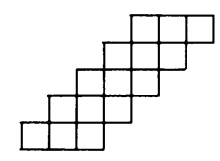

with all rows and all columns strictly increasing from left to right and from top to bottom, and with all entries in row $j$ at most $w-k+j$. This provides a surprising combinatorial interpretation of the Bernoulli number $B_{2 m}$ when $w=m+1$ and $k=m-1$ (in which case the top row of the staircase is forced to contain $1,2,3$ ).

The combinatorial interpretation proves in particular that $(-1)^{k} A_{k}^{(m)} \geq 0$ for all $k \geq 0$. Faulhaber stated this, but he may not have known how to prove it.

Denoting the determinant by $D(w, k)$, Jacobi's recurrence $(* *)$ implies that we have

$$
\begin{aligned}
& (w-k)^{2}(w-k+1)(w-k-1) D(w, k-1) \\
& =(2 w-2 k)(2 w-2 k-1)(w-k-1) D(w, k) \\
& \quad-2 w(2 w-1)(w-1) D(w-1, k)
\end{aligned}
$$

this can also be written in a slightly tidier form, using a special case of the "integer basis" polynomials discussed above:

$$
D(w, k-1)=T_{1}(w-k-1) D(w, k)-T_{1}(w-1) D(w-1, k) .
$$

It does not appear obvious that the determinant satisfies such a recurrence, nor that the solution to the recurrence should have integer values when $w$ and $k$ are integers. But, identities are not always obvious.

\section{Generalization to NONINTEGER POWERS}

Recurrence $(*)$ does not require $w$ to be a positive integer, and we can in fact solve it in closed form when $w=3 / 2$ :

$$
\begin{aligned}
\sum_{k \geq 0} A_{k}^{(3 / 2)} u^{3 / 2-k} & =B_{3}\left(\frac{\sqrt{1+4 u}+1}{2}\right) \\
& =\frac{u}{2} \sqrt{1+4 u}=u^{3 / 2} \sum_{k \geq 0}\left(\begin{array}{c}
1 / 2 \\
k
\end{array}\right)(4 u)^{-k}
\end{aligned}
$$

Therefore, $A_{k}^{(3 / 2)}=\left(\begin{array}{c}1 / 2 \\ k\end{array}\right) 4^{-k}$ is related to the $k$ th Catalan number. A similar closed form exists for $A_{k}^{(m+1 / 2)}$ when $m$ is any nonnegative integer.

For other cases of $w$, our generating function for $A_{k}^{(w)}$ involves $B_{n}(x)$ with noninteger subscripts. The Bernoulli polynomials can be generalized to a family of functions $B_{z}(x)$, for arbitrary $z$, in several ways; the best generalization for our present purposes seems to arise when we define

$$
B_{z}(x)=x^{z} \sum_{k \geq 0}\left(\begin{array}{l}
z \\
k
\end{array}\right) x^{-k} B_{k}
$$


choosing a suitable branch of the function $x^{z}$. With this definition we can develop the right-hand side of

$(* * *)$

$$
\begin{aligned}
\sum_{k \geq 0} A_{k}^{(w)} u^{-k} & =B_{2 w}\left(\frac{\sqrt{1+4 u}+1}{2}\right) u^{-w} \\
& =\left(\frac{\sqrt{1+4 u}+1}{2 \sqrt{u}}\right)^{2 w} \sum_{k \geq 0}\left(\begin{array}{c}
2 w \\
k
\end{array}\right)\left(\frac{\sqrt{1+4 u}+1}{2}\right)^{-k} B_{k}
\end{aligned}
$$

as a power series in $u^{-1}$ as $u \rightarrow \infty$.

The factor outside the $\sum$ sign is rather nice; we have

$$
\left(\frac{\sqrt{1+4 u}+1}{2 \sqrt{u}}\right)^{2 w}=\sum_{j \geq 0} \frac{w}{w+j / 2}\left(\begin{array}{c}
w+j / 2 \\
j
\end{array}\right) u^{-j / 2},
$$

because the generalized binomial series $B_{1 / 2}\left(u^{-1 / 2}\right)$ [5, equation $\left.(5.58)\right]$ is the solution to

namely

$$
f(u)^{1 / 2}-f(u)^{-1 / 2}=u^{-1 / 2}, \quad f(\infty)=1
$$

$$
f(u)=\left(\frac{\sqrt{1+4 u}+1}{2 \sqrt{u}}\right)^{2}
$$

Similarly we find

$$
\begin{aligned}
\left(\frac{\sqrt{1+4 u}+1}{2}\right)^{-k} & =\sum_{j} \frac{-k}{j-k}\left(\begin{array}{c}
j / 2-k / 2 \\
j
\end{array}\right) u^{-k / 2-j / 2} \\
& =u^{-k / 2}-\sum_{j \geq 1} \frac{k}{2 j}\left(\begin{array}{c}
j / 2-k / 2-1 \\
j-1
\end{array}\right) u^{-k / 2-j / 2}
\end{aligned}
$$

So we can indeed expand the right-hand side as a power series with coefficients that are polynomials in $w$. It is actually a power series in $u^{-1 / 2}$, not $u^{-1}$; but since the coefficients of odd powers of $u^{-1 / 2}$ vanish when $w$ is a positive integer, they must be identically zero. Sure enough, a check with computer algebra on formal power series yields $1+A_{1}^{(w)} u^{-1}+A_{2}^{(u)} u^{-2}+A_{3}^{(w)} u^{-3}+O\left(u^{-4}\right)$, where the values of $A_{k}^{(w)}$ for $k \leq 3$ agree perfectly with those obtained directly from (*). Therefore this approach allows us to express $A_{k}^{(w)}$ as a polynomial in $w$, using ordinary Bernoulli number coefficients:

$$
\begin{aligned}
A_{k}^{(w)}= & \sum_{l=0}^{2 k} \frac{w}{w+l / 2}\left(\begin{array}{c}
w+l / 2 \\
l
\end{array}\right) \times \\
& \times\left(\left(\begin{array}{c}
2 w \\
2 k-l
\end{array}\right) B_{2 k-l}-\frac{1}{2} \sum_{j=1}^{2 k-l-1}\left(\begin{array}{c}
2 w \\
j
\end{array}\right) \frac{j}{2 k-l-j}\left(\begin{array}{c}
k-l / 2-j-1 \\
2 k-l-j-1
\end{array}\right) B_{j}\right) .
\end{aligned}
$$

The power series $(* * *)$ we have used in this successful derivation is actually divergent for all $u$ unless $2 w$ is a nonnegative integer, because $B_{k}$ grows superexponentially while the factor

$$
\left(\begin{array}{c}
2 w \\
k
\end{array}\right)=(-1)^{k}\left(\begin{array}{c}
k-2 w-1 \\
k
\end{array}\right)=\frac{(-1)^{k} \Gamma(k-2 w)}{\Gamma(k+1) \Gamma(-2 w)} \sim \frac{(-1)^{k}}{\Gamma(-2 w)} k^{-2 w \prime-1}
$$


does not decrease very rapidly as $k \rightarrow \infty$. Still, $(* * *)$ is easily seen to be a valid asymptotic series as $u \rightarrow \infty$, because asymptotic series multiply like formal power series. This means that, for any positive integer $p$, we have

$$
\sum_{k=0}^{2 p}\left(\begin{array}{c}
2 w \\
k
\end{array}\right)\left(\frac{\sqrt{1+4 u}+1}{2}\right)^{2 w-k} B_{k}=\sum_{k=0}^{p} A_{k}^{(w)} u^{w-k}+O\left(u^{w-p-1}\right) .
$$

We can now apply these results to obtain sums of noninteger powers, as asymptotic series of Faulhaber's type. Suppose, for example, that we are interested in the sum

$$
H_{n}^{(1 / 3)}=\sum_{k=1}^{n} \frac{1}{k^{1 / 3}} .
$$

Euler's summation formula [5, Exercise 9.27] tells us that

$$
\begin{aligned}
H_{n}^{(1 / 3)}-\zeta\left(\frac{1}{3}\right) & \sim \frac{3}{2} n^{2 / 3}+\frac{1}{2} n^{-1 / 3}-\frac{1}{36} n^{-4 / 3}-\cdots \\
& =\frac{3}{2}\left(\sum_{k \geq 0}\left(\begin{array}{c}
2 / 3 \\
k
\end{array}\right) n^{2 / 3-k} B_{k}+n^{-1 / 3}\right),
\end{aligned}
$$

where the parenthesized quantity is what we have called $B_{2 / 3}(n+1)$. And when $u=n^{2}+n$, we have $B_{2 / 3}(n+1)=B_{2 / 3}((\sqrt{1+4 u}+1) / 2)$; hence,

$$
\begin{aligned}
H_{n}^{(1 / 3)}-\zeta\left(\frac{1}{3}\right) & \sim \frac{3}{2} \sum_{k \geq 0} A_{k}^{(1 / 3)} u^{1 / 3-k} \\
& =\frac{3}{2} u^{1 / 3}+\frac{5}{36} u^{-2 / 3}-\frac{17}{1215} u^{-5 / 3}+\cdots
\end{aligned}
$$

as $n \rightarrow \infty$. (We cannot claim that this series converges twice as fast as the classical series in $n^{-1}$, because both series diverge! But we would get twice as much precision in a fixed number of terms, by comparison with the classical series, except for the fact that half of the Bernoulli numbers are zero.)

In general, the same argument establishes the asymptotic series

$$
\sum_{k=1}^{n} k^{\alpha}-\zeta(-\alpha) \sim \frac{1}{\alpha+1} \sum_{k \geq 0} A_{k}^{((\alpha+1) / 2)} u^{(\alpha+1) / 2-k}
$$

whenever $\alpha \neq-1$. The series on the right is finite when $\alpha$ is a positive odd integer; it is convergent (for sufficiently large $n$ ) if and only if $\alpha$ is a nonnegative integer.

The special case $\alpha=-2$ has historic interest, so it deserves a special look:

$$
\begin{aligned}
\sum_{k=1}^{n} \frac{1}{k^{2}} \sim & \frac{\pi^{2}}{6}-A_{0}^{(-1 / 2)} u^{-1 / 2}-A_{1}^{(-1 / 2)} u^{-3 / 2}-\cdots \\
= & \frac{\pi^{2}}{6}-u^{-1 / 2}+\frac{5}{24} u^{-3 / 2}-\frac{161}{1920} u^{-5 / 2}+\frac{401}{7168} u^{-7 / 2} \\
& -\frac{32021}{491520} u^{-9 / 2}+\cdots
\end{aligned}
$$

These coefficients do not seem to have a simple closed form; the prime factorization $32021=11 \cdot 41 \cdot 71$ is no doubt just a quirky coincidence.

\section{ACKNOWLEDGMENTS}

This paper could not have been written without the help provided by several correspondents. Anthony Edwards kindly sent me a photocopy of Faulhaber's 
Academia Algebra, a book that is evidently extremely rare: An extensive search of printed indexes and electronic indexes indicates that no copies have ever been recorded to exist in America, in the British Library, or the Bibliothèque Nationale. Edwards found it at Cambridge University Library, where the volume once owned by Jacobi now resides. (I have annotated the photocopy and deposited it in the Mathematical Sciences Library at Stanford, so that other interested scholars can take a look.) Ivo Schneider, who is currently preparing a book about Faulhaber and his work, helped me understand some of the archaic German phrases. Herb Wilf gave me a vital insight by discovering the first half of Lemma 4, in the case $r=1$. And Ira Gessel pointed out that the coefficients in the expansion $n^{2 m+1}=\sum a_{k}\left(\begin{array}{c}n+k \\ 2 k+1\end{array}\right)$ are central factorial numbers in slight disguise.

\section{BIBLIOGRAPHY}

1. A. W. F. Edwards, A quick route to sums of powers, Amer. Math. Monthly 93 (1986), 451-455.

2. Johann Faulhaber, Academia Algebra, Darinnen die miraculosische Inventiones zu den höchsten Cossen weiters continuirt und profitiert werden, call number QA154.8 F3 1631a f MATH at Stanford University Libraries, Johann Ulrich Schönigs, Augspurg [sic], 1631.

3. Ira Gessel and University of South Alabama Problem Group, A formula for power sums, Amer. Math. Monthly 95 (1988), 961-962.

4. Ira M. Gessel and Gérard Viennot, Determinants, paths, and plane partitions, Preprint, 1989.

5. Ronald L. Graham, Donald E. Knuth, and Oren Patashnik, Concrete mathematics, Addison-Wesley, Reading, MA, 1989.

6. C. G. J. Jacobi, De usu legitimo formulae summatoriae Maclaurinianae, J. Reine Angew. Math. 12 (1834), 263-272.

7. John Riordan, Combinatorial identities, Wiley, New York, 1968.

8. L. Tits, Sur la sommation des puissances numériques, Mathesis 37 (1923), 353-355.

Department of Computer Science, Stanford University, Stanford, California 94305 